\title{
Tres discusiones acerca de la relación entre prueba y verdad
}

Diego Dei Vecchi

Recepción: 3/7/2013

Aceptación: 1/10/2013

\begin{abstract}
Resumen: El autor lleva a cabo un balance crítico de la discusión central de Discusiones 3 relativa a la prueba y verdad en el proceso judicial. En un primer apartado introductorio se reconstruye brevemente esa discusión introduciéndose las nociones de enunciado fáctico y razonamiento probatorio. A partir de allí se aborda la temática mediante una trifurcación, formulándose en cada ramal algunas críticas a las posturas presentadas en la discusión reconstruida. Primero, se analiza la relación entre la justificación del enunciado fáctico, su verdad y la justicia de la decisión. El segundo apartado trata acerca de la función, cognitiva o persuasiva, de la prueba judicial. Por último, se reconstruye la que se presenta como la cuestión central en la discusión contemporánea: la relativa a los estándares de prueba. El ensayo concluye mostrando el estado actual de discusión y conjeturando la orientación que ella habrá de adoptar en vistas a resolver ciertos inconvenientes teórico-prácticos.
\end{abstract}

Palabras clave: prueba judicial - enunciado probatorio - estándar de prueba - sistemas de valoración.

\section{Introducción: Enunciado fáctico y razonamiento probatorio}

El tercer número de Discusiones (2003) versaba sobre una cuestión que abandonaba lentamente su marginalidad en el ámbito jurídico filosófico: la relativa a la prueba y verdad de los enunciados fácticos de la 
decisión judicial ${ }^{1}$. Hasta entonces, o hasta muy poco antes, esos problemas no ocupaban un lugar central en la agenda de los juristas ni en la de los teóricos del derecho, al menos no en sus aristas epistemológicas 2 .

Posiblemente las razones de esa marginalización hayan de buscarse en concepciones del proceso judicial como procedimiento al cual, o bien le es indiferente tanto la verdad de los enunciados fácticos cuanto su justificación; o bien, aunque no siéndole indiferente la verdad de ellos, no ha de preocuparle especialmente su justificación como verdaderos, dado que se trataría de una cuestión externa, ya resuelta al momento de la decisión ${ }^{3}$.

Frente a estas concepciones, el enfático intento por dilucidar las cuestiones epistemológicas concernientes a la decisión judicial aparece a menudo ligado a ideologías especialmente preocupadas por la verdad de los enunciados fácticos en ellas contenidos conjugada con una visión más o menos optimista sobre la racionalidad epistémica como medio para la maximización del logro de ese fin, pero con una especial conciencia acerca de ciertas particularidades propias del ámbito jurídico ${ }^{4}$. Esta es la orientación de Discusiones 3, al momento de cuya aparición había comenzado algo tímidamente a proliferar, y

1 Andrés Ibáñez, Perfecto, "Sobre prueba y proceso penal", Discusiones, 3, 2003, p. 56. En adelante simplemente 'Andrés Ibáñez'. Esta omisión, por lo demás, en principio no habría sido tan grave en la cultura jurídica anglosajona (cfr. Anderson, Terence, William Twining \& David A. Schum, Analysis of evidence, New York, Cambridge University Press, 2005 [1991]).

2 El discurso de los procesalistas, se ha limitado en general a proporcionar una serie de distinciones relativas al término 'prueba' (i.e. elemento, objeto, procedimiento, medio, sujeto) y a describir o prescribir cuestiones concernientes a los distintos sistemas de valoración actuales o históricos, pero en general sin profundizar demasiado en sus implicancias epistémicas (cfr. por todos, Briseño Sierra, Humberto, Derecho procesal, México, D.F, Harla, 1995, p. 1360 y sgts, Couture, Eduardo J., Fundamentos de Derecho Procesal Civil, Buenos Aires, Depalma, 1958, p. 215-276).

3 Seguramente de allí el brocárdico que los procesalistas suelen poner en boca de los jueces: narra mihi factum, dabi tibi ius.

${ }^{4}$ Como decía Guastini al prologar Ferrer Beltrán, Jordi, La valutazione razionale della prova, Milano, Giuffrè, 2012: una 'concepción iluminista' de la prueba. 
Tres discusiones acerca de la relación entre prueba y verdad

luego proliferó exponencialmente, la literatura con este enfoque.

No es casual que el debate se haya planteado alrededor de un texto de Michele Taruffo5: uno de los artífices de este 'giro epistemológico' en el tratamiento de la prueba jurídica; giro que ha consistido en buena medida en el trazado de puentes entre epistemología, derecho procesal y filosofía del derecho ${ }^{6}$; todo lo cual queda plasmado en las réplicas a cargo de Marina Gascón Abellán ${ }^{7}$, Perfecto Andrés Ibáñez y Andrés Bouzat y Alejandro Cantaro ${ }^{8}$.

El objetivo de este texto es ofrecer un mapa de ese debate, reconsiderando tres cuestiones salientes a la luz de la literatura que comenzaba a proliferar en la materia e intentado proporcionar, en definitiva, un panorama lo más amplio posible del, como suele decirse, 'estado del arte'.

En términos meramente introductorios, ocupaban un lugar central en Discusiones 3, la cuestión acerca de la relación entre verdad del enunciado fáctico y justificación de la decisión judicial (¿es la verdad o la prueba del enunciado fáctico la condición de justificación de la decisión judicial?), el papel o función de la prueba en relación a dicho enunciado (¿es la prueba cognición o persuasión?) y la relevancia de la

5 TARUfFo, Michele, "Algunas consideraciones sobre la relación entre prueba y verdad", Discusiones, 3, 2003. En adelante Taruffo ${ }_{a}$.

6 En efecto, TARUfFo, Michele, La prova dei fatti giuridici: nozioni generali, Milano, Giuffre, 1992 es considerado generalizadamente como una obra cardinal en esta orientación. Recientemente decía Andrés Ibáñez que Taruffo encarna, en este sentido, a un "procesalista felizmente atípico, trufado de epistemólogo y de teórico y filósofo del derecho, que ha tenido la audacia intelectual de adentrarse en un campo minado de dificultades» (prólogo a Taruffo, Michele, Perfecto Andrés Ibáñez \& Alffonso Candau Pérez, Consideraciones sobre la prueba judicial, Madrid, Fundación Coloquio Jurídico Europeo, 2010, p. 11).

7 Gascón Abellán, Marina, "Concepciones de la prueba. Observación a propósito de Algunas consideraciones sobre la relación entre prueba y verdad, de Michele Taruffo", Discusiones, 3, 2003. En adelante 'Gascón Abellán'.

8 Bouzat, Andrés \& Alejandro S. CANTARo, "Verdad y prueba en el proceso acusatorio", Discusiones, 3, 2003. En adelante 'Bouzat\&Cantaro'. A todas las réplicas contestaba unificadamente TARUfFo, Michele, "Algunos comentarios sobre la valoración de la prueba", Discusiones, 3, 2003. En adelante, Taruffo ${ }_{b}$. 


\section{Diego Dei Vecchi}

inmediación entre decisor y prueba (¿puede solo valorar correctamente quien tenga relación de inmediación con la prueba?).

Pues bien, Taruffo iniciaba la discusión haciendo algunas consideraciones acerca de los enunciados fácticos de la decisión judicial. Ubicaba a la justificación de esta clase de enunciados, como es usual, dentro de la que suele denominarse 'justificación externa' de la decisión: la justificación independiente de cada una de las premisas (i.e. la mayor, o norma general y abstracta; y la menor, o enunciado fáctico) cuya conjunción habilitaría, por modus ponens, el paso a la conclusión (i.e. la 'norma individual') $)^{9}$.

La norma general y abstracta que constituye la premisa mayor sería producto de un proceso interpretativo-argumentativo complejo que no será tratado aquí, pero que evocando a Guastini podría sintetizarse como aquél que va 'de la disposición a la norma'10. La correcta interpretación, decía Taruffo, es una de las condiciones que debe satisfacer la decisión para ser justa.

Por su parte, los enunciados fácticos (i.e. las premisas menores), serían enunciados declarativos tales que «pueden ser verdaderos o falsos; tienen como objeto circunstancias que, aunque puedan contener implicaciones valorativas y (...) son en alguna manera, social y culturalmente "construidas", pueden darse o no darse en el mundo de las experiencias concretas» ${ }^{11}$. Taruffo sostenía que estos enunciados se construyen en al menos cuatro sentidos: selectivo, semántico, cultural y social.

La construcción selectiva implicaría elegir ciertas circunstancias de la realidad especialmente relevantes en una situación concreta y con determinada finalidad. Esta selección estaría guiada por (al menos) dos clases de criterios de relevancia: los jurídicos y los lógicos.

Los criterios jurídicos llevarían a seleccionar dentro de las múlti-

9 Esto último (i.e. el paso de las premisas a la conclusión por modus ponens) constituye la llamada 'justificación interna' de la decisión judicial. Cfr., por todos, WRÓBLEWSKI, JERZY, "Il modello teorico dell'applicazione della legge", Rivista internazionale di Filosofia del Diritto, 46, 1967.

${ }^{10}$ Por todos, GuAstini, Riccardo, Interpretare e argomentare, Milano, Giuffrè, 2011, en especial cap. IV.

${ }^{11}$ Taruffo $_{\mathrm{a}}$, p. 15. 
Tres discusiones acerca de la relación entre prueba y verdad

ples descripciones posibles de la realidad, aquellas que muestran al enunciado fáctico como instancia del supuesto de hecho de la norma constituyente de la premisa mayor. Esto no es más que aquello que muchos autores denominan 'razonamiento calificatorio', por contraposición con el 'razonamiento probatorio'"12.

Los criterios lógicos, por su parte, apuntarían a «aquellos hechos que no son jurídicamente calificados por norma alguna, pero que, sin embargo, pueden entrar en el proceso en la medida en que a través de su conocimiento se pueden extraer conclusiones útiles para demostrar la verdad o falsedad de un hecho jurídicamente calificado» ${ }^{13}$. La relevancia de estos (enunciados sobre) hechos estaría, según el autor, en el proporcionar sustento inferencial al enunciado fáctico construido según los criterios de relevancia jurídica. De allí que los denomine (enunciados sobre) hechos indiciarios.

Pues bien, y a modo de primer comentario, no parece riguroso considerar a los (enunciados sobre) hechos indiciarios 'criterios de construcción' del enunciado fáctico. Ellos son, más precisamente, elementos de la inferencia que le da apoyo probatorio a ese enunciado 'una vez construido' (i.e. ya calificado); se trata de las premisas del razonamiento probatorio o más simplemente, de las pruebas.

Sobre esto nos centraremos a continuación, especialmente en relación a tres cuestiones: Primero, la relativa a si la verdad del enunciado

${ }^{12}$ La «traducción al lenguaje jurídico» (WRÓBLEWSKI, JERZY, "Il modello teorico dell'applicazione della legge", Rivista internazionale di Filosofia del Diritto, 46, 1967, p. 18). Sobre esta clase de problemas, por todos, Ferrua, PaOlo, Il giudizio penale: fatto e valore giuridico, en A.A.V.V., La prova nel dibattimento penale, Torino, G. Giappichelli, 317-410, 2007, GonzÁlez Lagier, Daniel, Quaestio facti: ensayos sobre prueba, causalidad y acción, Lima-Bogotá, PalestraTemis, 2005, González Lagier, Daniel, "Hechos y conceptos", Dialnet: cuadernos electrónicos de filosofia del derecho, 15, 2007, MazzArese, Tecla, Forme di razionalità delle decisioni giudiziali, Torino, Giappichelli, 1996, p. 87, Ubertis, GiULIo, Fatto e valore nel sistema probatorio penale, Milano, Giuffrè, 1979. Dejaré de lado en esta presentación las cuestiones concernientes al razonamiento calificatorio, sobre todo porque no han sido centrales en la discusión que aquí se reconsidera.

${ }^{13}$ Taruffo $_{\mathrm{a}}$, p. 19. 


\section{Diego Dei Vecchi}

fáctico es condición necesaria de la justificación de la decisión judicial y, en su caso, cuál es el papel del razonamiento probatorio a tal efecto (§1). Segundo, la relativa a la concepción de la prueba dentro del proceso ( $\$ 2.1)$, y la consecuente relevancia atribuida a la 'inmediación' (\$2.2), de acuerdo al modo en que se entienda la relación entre premisas probatorias y enunciado fáctico. Por último, la relativa a los estándares de prueba, como criterios determinativos de la suficiencia de las pruebas en favor del enunciado fáctico de la decisión (§3).

\section{Primera discusión: justificación de enunciados fácticos, verdad y decisión justa}

Las condiciones de justificación de los enunciados fácticos de la decisión judicial parecen de algún modo determinadas por la concepción que se tenga del proceso judicial y la finalidad que correspondientemente se le asigne. Taruffo planteaba en este aspecto dos alternativas como disyuntivas: (i) concebir al proceso como método de resolución de conflictos dirigido a poner término a la disputa; (ii) concebir al proceso como método para «aplicar la ley, poner en práctica el derecho y garantizar efectivamente los derechos individuales y colectivos...» ${ }^{14}$.

La primera opción identificaría la justicia de la decisión como meramente procedimental: mero "respeto de las reglas del juego». Taruffo apuntaba principalmente al denominado adversary system, un proceso, basado en una ideología para la cual la verdad es irrelevante, entendido como «competición deportiva» donde «no gana quien tiene razón; tiene razón quien gana» ${ }^{15}$.

De conformidad con la segunda visión, «la justicia del procedimiento puede considerarse, ciertamente, como una condición de justicia de la decisión (al menos en el sentido de que no estamos dispuestos

\footnotetext{
${ }^{14}$ Taruffo $_{\mathrm{a}}, \mathrm{p} 26$.

${ }^{15}$ TARUfFO, Michele, La semplice verità: il giudice e la costruzione dei fatti, Bari, Laterza, 2009, p. 109. El sistema adversarial ha sido definido como aquel en el cual el proceso está bajo el control de la partes y el órgano decisor resta esencialmente pasivo (DAMAšKa, MirJAn R., Il Diritto delle prove alla deriva, Bologna, il Mulino, 2003, p. 109).
} 
a aceptar como justa una decisión tomada dentro de un proceso injusto, en el cual, por ejemplo: el juez no practicó pruebas o hubiese decidido tirando los dados o basándose en una confesión obtenida con la tortura), pero no puede ser considerada como la única condición, o como condición suficiente de la justicia de la decisión» ${ }^{16}$.

Para Taruffo, la justicia del resultado del proceso (i.e. de la decisión) dependería no solo del respeto de las 'reglas del juego' sino de «criterios substanciales de justicia» que identifica con la correcta «aplicación de normas en los casos concretos que son objeto de decisión» ${ }^{17}$. Esta «correcta aplicación de normas», por su parte, se daría solo si (a) la norma es adecuada para el caso y ha sido correctamente interpretada, y si (b) se ha determinado la verdad acerca de los hechos que constituyen el caso ${ }^{18}$.

Para Taruffo «es evidente que si la norma $\mathrm{N}$ identifica el supuesto de hecho $\mathrm{H}$ como premisa necesaria para determinados efectos jurídicos, pero el hecho individual $h$ que corresponde a $\mathrm{H}$ no existe (en la medida en que es falso el enunciado que lo describe), entonces la norma $\mathrm{N}$ no puede ser aplicada en este caso. Si, de todas manera, el juez la aplica, ello basta para decir que la decisión no es justa» ${ }^{19}$. El autor basa esta afirmación en que: «es obvio que a un proceso justo puede seguir una decisión injusta, si el juez se equivoca al interpretar la norma o si los hechos no se determinan correctamente porque el juez valora en modo equivocado el resultado de las pruebas» ${ }^{20}$.

No obstante, si se asume (como parece plausible) que las reglas de valoración de la prueba forman parte del procedimiento, pues entonces una incorrecta valoración de la prueba como la de la cita precedente, produce una situación de 'injusticia procedimental', una violación de 'las reglas del juego', solapada por lo tanto con la 'injusticia substancial' que preocupa a Taruffo. El caso interesante para

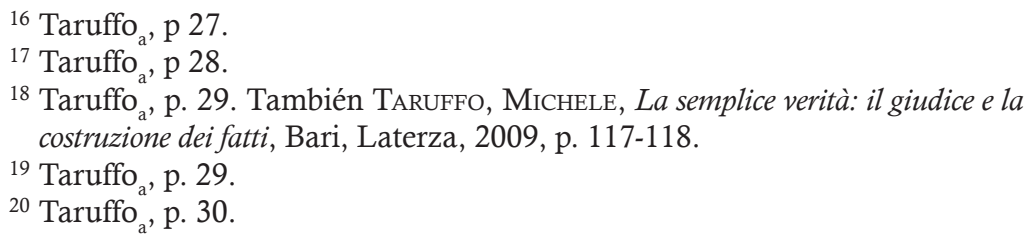




\section{Diego Dei Vecchi}

contraponer 'justicia procedimental' y 'justicia substancial', por el contrario, parece ser más bien aquél en que las reglas de valoración son respetadas, de modo que se declara correctamente probado (o no probado) el enunciado fáctico falso (o verdadero): ¿está allí jurídicamente justificada la decisión?, ¿en su caso, es también justa?

Parece haber aquí una ambigüedad en la posición de Taruffo desde que la condición (b) de 'aplicación correcta de normas' (i.e. la 'determinación de la verdad') podría interpretarse según sus palabras, ya como (i) verdad del enunciado a secas (i.e. correspondencia con la realidad) ${ }^{21}$, ya como (ii) prueba de la verdad del enunciado (i.e. aparente correspondencia dadas las pruebas valoradas) ${ }^{22}$.

A esto apuntaban especialmente Bouzat y Cantaro en su réplica, basándose en algunas ideas de Ricardo Caracciolo centradas en la ambigüedad de la noción de 'decisión judicial' que, como es sabido, designa tanto la norma individual (conclusión de la justificación interna de la decisión), cuanto la acción de decidir. Correlativamente, cabe distinguir entre justificación de la acción y justificación de su resultado (i.e. de la norma individual): «[1]a justificación es una noción relacional, que hace referencia a la relación existente entre un dominio de

${ }^{21}$ Así interpretan Bouzat\&Cantaro la postura de Taruffo, en el sentido «... de que solo constituye una decisión judicial justa aquella que se funda en premisas fácticas verdaderas...» (Bouzat\&Cantaro, p. 70). Esta interpretación encuentra sustento en algunas afirmaciones de Taruffo, por ejemplo: si el hecho «no se ha producido "en la realidad" no se puede aplicar la norma y, si se aplica, es suficiente para calificar como injusta la sentencia» (Taruffo ${ }_{a}$, p. 39).

${ }^{22}$ Son varios los pasajes donde la posición del autor se torna ambigua, por ejemplo: «... un enunciado fáctico es verdadero si está confirmado por pruebas y es falso si las pruebas disponibles confirman su falsedad; y no está probado si en el proceso no se adquirieron pruebas suficientes para demostrar su verdad o falsedad» (Taruffo ${ }_{a}$, p. 31, negritas propias); «la concepción de la verdad procesal como correspondencia con la realidad externa y, por lo tanto, como demostrabilidad en juicio mediante pruebas, es válida para todo tipo de hecho que el juez deba verificar» (Taruffo, p. 40, cursivas propias); «[1]o que persigue el proceso es la mejor aproximación posible, basada en las pruebas que estén disponibles en el caso proceso concreto, a la verdad "histórica" o "empírica" de los hechos» (Taruffo, , p. 86, cursivas propias). 
razones y un contradominio que hay que justificar (normas, acciones, creencias, proposiciones, etc.) $\rangle^{23}$. Y en efecto, la aparente ambigüedad en la posición de Taruffo respecto a las condiciones de justificación de la premisa fáctica, se corresponde con la presencia de estas dos decisiones (i.e. acción y norma) a justificar.

En este sentido, es usual decir que una norma estará justificada en tanto sea aplicación, i.e. implicación lógica, de otra norma. Para Caracciolo, aplicación de normas implica la verdad de los enunciados fácticos subsumibles en el supuesto de hecho de la norma aplicada; luego, la condición de justificación de la decisión-norma es la verdad del enunciado fáctico ${ }^{24}$.

Por el contrario, dado que no hay relaciones lógicas entre normas y acciones, la justificación de la acción de decisión del juez no podría darse por implicación lógica. Tal acción estaría justificada, en cambio, si fuere llevada a cabo en las circunstancias bajo las cuales una norma la ordena o faculta. Y parece evidente que los ordenamientos procesales ordenan o facultan a los jueces a decidir (i.e. a emitir normas individuales) en relación a determinadas normas generales 'si los enunciados fácticos en estas últimas subsumibles están probados'; luego, la condición de justificación de la decisión-acto es la prueba del enunciado fáctico.

Así las cosas, se daría entonces la paradoja de que el ordenamiento jurídico consideraría justificadas (ordenaría o facultaría) decisiones acto consistentes en la emisión de decisiones norma injustificadas (i.e. no 'aplicativas' de normas generales). Esta situación se daría en todos los casos en que los enunciados fácticos relevantes estuviesen probados siendo, sin embargo, falsos.

Ahora bien, dejando por un momento de lado la posición de Taruffo sobre el punto, ¿qué sería lo paradójico de esta situación: que el dere-

${ }^{23}$ Bouzat\&Cantaro, p. 69. CARACCIOLO, RicARDO A., Justificación normativa y pertenencia, en CARACCIOLO, RicARdo A., El derecho desde la filosofía: ensayos, Madrid, Centro de Estudios Políticos y Constitucionales, 37-69, 2009, p. 43.

${ }^{24}$ CARACCIOLO, RicARDo, El problema de los hechos en la justificación de la sentencia, Universidad Nacional del Sur, 2000. 
cho pueda conducir a resultados injustos mediante decisiones (jurídicamente) justificadas (por declarar probados enunciados fácticos falsos); o bien que una descripción del derecho lo muestre como incoherente (por darse en su interior, según sus criterios internos, acciones jurídicamente justificadas con resultados injustificados)? Porque al tiempo que no parece haber nada de paradójico en lo primero, consecuencia de la falibilidad de todo método de decisión no omnisciente; si el problema fuere del segundo tipo, la paradoja pareciera disolverse con una reformulación de los criterios jurídicos de justificación.

En este sentido, Sánchez Brígido y Seleme proponían hace algunos años enlazar los criterios de justificación de los actos de decisión con los de las normas resultantes reconstruyendo «la norma que justifica el acto como haciendo referencia a la justificación de su contenido» ${ }^{25}$. De este modo, si 'aplicación correcta de normas' implica conceptualmente (i) la verdad de los enunciados fácticos relevantes, habrá que incluir entre las condiciones de permisión o mandato del acto de decisión judicial el que esos enunciados sean verdaderos. Por el contrario, si 'aplicación correcta de normas' requiere solamente (ii) la prueba de los enunciados fácticos (sean verdaderos o falsos), pues entonces se podrá considerar sin problemas también a la mera prueba de los enunciados como la condición justificativa del acto de decisión. De este modo, decisión norma y decisión acto estarán justificados o injustificados en los mismos casos, al menos a la luz del derecho.

Recientemente, optando aparentemente por la segunda opción ${ }^{26}$, decía Taruffo que la justicia de la decisión «no presupone solamente su legalidad, es decir su derivación de una interpretación y aplicación correcta de las normas sino también su veracidad, es decir la comprobación de la verdad de los hechos relevantes...» ${ }^{27}$. Pero si esto es así, pues

${ }^{25}$ Sánchez Brígido, Rodrigo \& Hugo Oscar Seleme, "Justificación sin verdad", Analisi e diritto 2001, 2002, p. 292.

${ }^{26}$ Véanse además las citas en nota 22.

27 Taruffo, Michele, Consideraciones sobre prueba y motivación, en Taruffo, Michele, Perfecto Andrés Ibáñez \& Alfonso Candau Pérez, Consideraciones sobre la prueba judicial, Madrid, Fundación Coloquio 
entonces lo que el autor llama justicia substancial y justicia procedimental colapsan: la realización correcta del procedimiento, incluida la valoración de la prueba, satisface las condiciones de justicia substancial. De hecho, Taruffo afirmaba metafóricamente que puede no llegarse al polo sur, «pero si quiero ir a la Tierra del Fuego debo saber en qué dirección moverme» ${ }^{28}$. El punto es, precisamente, que donde se tiene la finalidad de llegar es a Tierra del Fuego, no al polo sur: a la prueba de los hechos y no (necesariamente) a su verdad.

De este modo, además, las dos concepciones procesales presentadas como antagónicas se acercan considerablemente: no hay inconvenientes en concebir al proceso judicial como 'método de resolución de conflictos' ya que su realización correcta garantiza la 'correcta aplicación de las normas jurídicas'.

Así vista la cuestión, la relación entre prueba y verdad habría de analizarse en diversos niveles. En primer lugar, preguntándose si el proceso judicial debe estar dirigido a pronunciamientos con enunciados fácticos verdaderos. En este sentido, se han dado buenas razones para mostrar que de ello depende el éxito del derecho mismo en su función de dirección de la conducta de los ciudadanos ${ }^{29}$. En segundo lugar, asumiendo que así debe ser, cabría preguntarse todavía si los procesos judiciales tal como se estructuran efectivamente tienen en vistas satisfacer tal exigencia normativa, si es como debe ser. En tercer lugar, cabría preguntarse también si esas reglas son respetadas en cada sistema procesal y en su caso en qué grado, si son eficaces en vistas a alcanzar la verdad, etc. ${ }^{30}$.

El que un determinado ordenamiento procesal esté o no interesado en descubrir la verdad y, en su caso, cuán interesado esté, parece ser función, entre otras cosas, de las exigencias de pruebas epistémica-

Juridico Europeo, 17-46, 2010, p. 28. Cursivas del autor.

${ }^{28} \mathrm{Taruffo}_{\mathrm{b}}$, p. 86 .

${ }^{29}$ Se habla, en este sentido, de la verdad como ideal regulativo y de relación teleológica entre prueba y verdad: por todos, Ferrer Beltrán, Jordi, $\mathrm{La}$ valoración racional de la prueba, Madrid, Marcial Pons, 2007, p. 29-30.

30 Taruffo, Michele, Consideraciones sobre prueba y motivación, en Michele Taruffo, Perfecto Andrés Ibáñez \& Alfonso Candau Pérez, Consideraciones sobre la prueba judicial, Madrid, Fundación Coloquio Jurídico Europeo, 17-46, 2010, p. 25. 
mente relevantes para adoptar la decisión final, de cuánta prueba se exija para considerar que los enunciados fácticos están probados y de quién deba o pueda ofrecer y producir prueba ${ }^{31}$. Estas cuestiones son objeto de discusión todavía hoy.

En lo que aquí importa, cabe decir solamente que si el ordenamiento exige efectivamente que la decisión se base en pruebas genuinamente epistémicas, a lo sumo, quedará por discutirse si la medida en que esa regulación propende a descubrir la verdad es o no satisfactoria, si se tutelan o no suficientemente otros valores, etc.; pero no cabrá decir que no le interesa la verdad sin más por el solo hecho de concebirse al proceso como adversarial ${ }^{32}$.

${ }^{31}$ Si bien no puede aquí abordarse la cuestión en detalle, suele darse gran importancia al rol que el ordenamiento procesal reserva al juzgador como indicador de su preocupación por la determinación de la verdad de los enunciados fácticos. Para Taruffo un juez 'árbitro' sin poderes probatorios (como el del procedimiento adversarial) lleva a un proceso donde 'gana el más fuerte' sin ser relevante si el enunciado fáctico sobre el que se decide es verdadero o no. Frente a él, un juez con un rol de 'gobierno del proceso' y poder probatorio autónomo, sería garante de la 'correcta aplicación de la ley' lo que propendería a enunciados verdaderos (Taruffo ${ }_{b}$, p. 34-35). Parte de la objeción de Bouzat\&Cantaro apunta a esta visión que está en clara contradicción con la de aquellos que entienden al contradictorio entre dos iguales frente a un tercero neutral precisamente como garantía epistemológica (por todos, Ferrajoli, Luigi, Derecho y razón: teoría del garantismo penal, Madrid, Trotta, 2005, p. 152 y Redondo, MARÍA CRistina, "Argumentación auténtica y argumentación estratégica", Revista Brasileira de Filosofia, 238 1, 2012, p. 39, nota 17). Estas dos posturas llevan a los procesalistas a muy diversas conclusiones en temas como el de las 'cargas dinámicas de la prueba', las 'medidas para mejor proveer', etc. Parte de esta discusión puede verse en VARGAS, ABRAHAM LUIS, "Cargas probatorias dinámicas, sus perfiles actuales y algunas respuestas para sus críticos", Activismo y garantismo procesal: Ediciones de la Academia Nacional de Derecho y Cs. Sociales de Córdoba, XL 7, 2009.

${ }^{32}$ De allí también que sea sospechosa la pretendida conexión necesaria entre el proceso adversarial con la 'prueba como persuasión' (idea presente en Gascón Abellán, p. 54) ya que si el juez (aun siendo completamente neutral y probatoriamente pasivo) debe decidir de acuerdo a las pruebas que se ofrecieron y produjeron, y si ha de hacerlo de acuerdo a un criterio de suficiencia con bases epistémicas, entonces nada obsta a que decida declarando ganador a quien tenga buenas o mejores razones epistémicas. Por cierto, puede dudarse de que el adversary system, resulte más o menos idóneo 
Tres discusiones acerca de la relación entre prueba y verdad

Por lo que queda, asumiendo que son desde algún punto de vista injustas las normas individuales dictadas en base a enunciados fácticos falsos, será entonces inevitable reconocer que el derecho otorga 'definitividad' (y que considera acaso justificadas) a decisiones (acto y norma) injustas ${ }^{33}$. Exactamente del mismo modo, el derecho nunca se ha visto impedido de otorgar definitividad y considerar jurídicamente justificadas decisiones (acto y norma) que pueden afirmarse como injustas aun cuando basadas en premisas fácticas verdaderas. Esto es así toda vez que lo injusto sean las normas jurídicas correctamente aplicadas. En cualquiera de los casos, no encuentro nada paradójico en afirmar que el derecho fue, es, o podrá ser injusto.

\section{Segunda discusión: la prueba judicial, entre conoci-}

para el descubrimiento de la verdad, así como también, y sobre todo, de si ese tipo de procedimiento es justo en términos sociales; pero estas son dos cuestiones muy distintas (sobre lo primero puede verse, entre otros, DAmašKa, MirJan R., Il Diritto delle prove alla deriva, Bologna, il Mulino, 2003, p. 137-150, Fiss, Owen M., "The Social and Political Foundations of Adjudication", Law and Human Behavior, 62 , 1982).

${ }^{33}$ Esto podría presentarse de varios modos según cómo se combinen las condiciones normativas que justifican la decisión (acto y norma), de conformidad a la propuesta antes reproducida. Si se asumiese que decisión acto y norma están justificadas si los enunciados fácticos están probados, habrá decisiones jurídicamente justificadas pero extrajurídicamente injustas toda vez que los enunciados fácticos probados fueren falsos. Si, por el contrario, se asumiese que decisión acto y norma solo están justificadas si los enunciados fácticos son verdaderos, habrá decisiones injustas toda vez que el derecho otorgue (quizás paradójicamente) carácter definitivo a decisiones que, por ser falsos sus enunciados fácticos, están (incluso jurídicamente) injustificadas. Esta última alternativa ha llevado a marcar una analogía entre este fenómeno y la cláusula alternativa tácita kelseniana (Ferrer Beltrán, Jordi, 'Está probado que p', en Triolo, Lucia, Prassi giuridica e controllo di razionalità, I, Torino, G. Giappichelli, 73-96, 2001, p. 76). 


\section{miento y persuasión}

Taruffo traía a colación dos concepciones contrapuestas de la prueba judicial: prueba como conocimiento y prueba como persuasión ${ }^{34}$. Marina Gascón Abellán apuntaba especialmente a este fragmento, conectando cada una de esas concepciones con ciertas posturas epistemológicas: (a) objetivista crítica (identificada con la prueba como conocimiento) ${ }^{35}$; (b) constructivista (identificada con la prueba como persuasión) ${ }^{36}$; y (c) cognoscitivista acrítica ${ }^{37}$.

\section{II.1. Persuasión y verdad}

Decía Taruffo, y reproducía Gascón Abellán, que «una concepción de este tipo [de la prueba como medio de persuasión] es compatible con (y, más aún, implica) una concepción irracional de la valoración de la prueba» ${ }^{38}$. Se atribuía a la concepción persuasiva el hacer colap-

34 También, Taruffo, Michele, Consideraciones sobre prueba y motivación, en Michele Taruffo, Perfecto Andrés Ibáñez \& Alfonso Candau Pérez, Consideraciones sobre la prueba judicial, Madrid, Fundación Coloquio Jurídico Europeo, 17-46, 2010, p. 30.

${ }^{35}$ Lo que implicaría asumir que hay 'hechos externos' susceptibles de ser conocidos, que las pruebas son instrumentos para ello y que, en cualquier caso, el conocimiento es falible (Gascón Abellán, p. 44 y Taruffo a p. 32).

${ }^{36}$ Aquí se «entiende que la objetividad del conocimiento deriva de nuestros esquemas de pensamiento y juicios de valor, es decir, la verdad de los enunciados está muy vinculada al contexto» (Gascón Abellán, p. 45).

${ }^{37} \mathrm{El}$ cual «entiende que el fin (un fin instrumental) del proceso es averiguar la verdad de las aserciones litigiosas (en el sentido de su correspondencia con los hechos que describen), pero, al concebir la prueba judicial como un proceso guiado por reglas más o menos seguras, confía en la posibilidad de obtener resultados incuestionables» (Gascón Abellán, p. 47). Estas relaciones habían sido ya exploradas en GASCÓN ABELLÁN, MARINA, Los hechos en el derecho: Bases argumentales de la prueba, Madrid-Barcelona, Marcial Pons, 2004, Cap. II.

${ }^{38}$ Gascón Abellán, p. 45 y Taruffo a p. 32. En el mismo sentido, la prueba como persuasión «... se sitúa fuera de toda perspectiva racional ya que toma en cuenta solo la función persuasiva de la prueba...» (TARUFFO, Michele, Consideraciones sobre prueba y motivación, en Michele TARUfFo, Perfecto Andrés IbáÑEz \& 
Tres discusiones acerca de la relación entre prueba y verdad

sar confirmabilidad y verdad, de modo que verdadero sería aquello, y solo aquello, «que resulta probado en el proceso ${ }^{39}$. En este sentido, si probado en el proceso significase corazonada o convicción irracional del decisor, entonces la verdad sería el contenido proposicional de la corazonada o convicción irracional.

Así presentadas, las ideas de prueba y de verdad resultan claramente contra-intuitivas. Sin embargo, parece posible matizar el pretendido enlace necesario entre prueba como persuasión y valoración irracional de la prueba, así como también su incompatibilidad con el concepto de verdad como correspondencia.

En primer lugar, en el lenguaje ordinario persuasión significa «aprehensión o juicio que se forma en virtud de un fundamento»; y persuadir significa «inducir, mover, obligar a alguien con razones a creer o hacer algo» ${ }^{40}$. Así concebida, por definición, la persuasión implica algún criterio de corrección ${ }^{41}$. Y como consecuencia de ello, no cualquier cosa, no «cualquier causa ${ }^{42}$, podrían llevar a la persuasión del juez, sino tan solo aquellas que fundamenten su juicio de conformidad con ese criterio ${ }^{43}$.

Alfonso Candau Pérez, Consideraciones sobre la prueba judicial, Madrid, Fundación Coloquio Jurídico Europeo, 17-46, 2010, p. 31).

${ }^{39}$ Gascón Abellán, p. 46.

${ }^{40}$ Diccionario de la Real Academia Española.

${ }^{41} \mathrm{La}$ posición contraria que aquí se evalúa no parece del todo ajena al pensamiento de Taruffo quien afirma en un pasaje que la prueba como persuasión se dirige a convencer al juzgador «de lo fundado o infundado de un enunciado fáctico» (Taruffo ${ }_{a}$, p. 32).

${ }^{42}$ Taruffo $_{\mathrm{a}}$, p. 33.

${ }^{43}$ En este sentido, es perfectamente posible distinguir la 'persuasión' del juez 'fundada' en el ofrecimiento de dádivas por parte de una de las partes o sus representantes, de la 'persuasión' del juez 'fundada' en aquellas que Taruffo llama pruebas en sentido estricto (Taruffo a, p. 30). De allí que parezca incorrecto también inferir del hecho de que la prueba frente al jury norteamericano es persuasión, y de que éste nunca motiva su veredicto, que éste "por lo tanto, queda siempre falto de todo fundamento racional» (TARUfFO, Michele, Consideraciones sobre prueba y motivación, en Michele Taruffo, Perfecto Andrés Ibáñez \& Alfonso Candau Pérez, Consideraciones sobre la prueba judicial, Madrid, Fundación Coloquio 


\section{Diego Dei Vecchi}

En segundo lugar, ¿en qué puede consistir esa corazonada o esa convicción -por irracional que fuere- si no es precisamente en la correspondencia de la proposición expresada por el enunciado fáctico (y la creencia o corazonada) con la realidad ${ }^{44}$ Taruffo decía que la visión de la prueba como persuasión no es «totalmente falsa». Ella sería correcta desde el punto de vista «absolutamente parcial» del abogado, «quien usa las pruebas no con el fin de conocer o de comunicar conocimiento, sino con el fin de convencer al juez sobre la credibilidad de su versión de los hechos» ${ }^{45}$. Pero ¿qué puede significar 'convencer sobre su credibilidad' sino en el sentido de correspondencia con la realidad?

El problema con 'la mera persuasión', entendida en el sentido restringido de simple convicción, 'casi corazonada', no parece estar tanto en ella misma como criterio probatorio sino en su relación con ciertos motivos que podrían explicar la presencia de ese estado subjetivo en el decisor y que quedarían inmunes al control ${ }^{46}$. Desatender esos motivos conduciría a ciertos resultados insatisfactorios por 'sobre' o 'infra-incluyentes' en relación a las razones epistémicas con que eventualmente se cuente. Lo primero, en los casos en que el decisor estuviere 'persuadido' (por tanto, esté probado el enunciado) siendo insuficientes las razones

Juridico Europeo, 17-46, 2010, p. 24 resaltado propio). Motivar es explicitar razones, pero de la ausencia de (necesidad de) explicitación no se sigue ausencia de razones.

${ }^{44}$ Este punto era ya señalado en Ferrer Beltrán, Jordi, Prueba y verdad en el derecho, Madrid, Marcial Pons, 2002, p. 79.

${ }^{45}$ Taruffo $_{\mathrm{a}}$, p. 33.

${ }^{46}$ Por ejemplo, por 'fundarse' en argumentos 'persuasivos' pero falaces, etc. Este temor está fundado seguramente por razones históricas (por todos, Couture, Eduardo J., Fundamentos de Derecho Procesal Civil, Buenos Aires, Depalma, 1958, p. 273, Ferrajoli, Luigi, Derecho y razón: teoría del garantismo penal, Madrid, Trotta, 2005, p. 139, MaIER, Julio B., Derecho Procesal Penal: Fundamentos, 1, Buenos Aires, del Puerto, 2004, p. 445 y sgts.). Más recientemente Taruffo mostraba a la intima convicción en dos facetas diversas, una pervertida por irracional y otra conducente a y coincidente con la aplicación de las 'reglas de la razón' (TARUfFo, Michele, La semplice verità: il giudice e la costruzione dei fatti, Bari, Laterza, 2009, p. 161). Ver también ENGEL, CHRISTOPH, "A behavioral perspective on a conflict between american and continental european law", Vermont Law Review, 33, 2009. 
epistémicas disponibles ${ }^{47}$. Lo segundo, cuando él no se sintiere persuadido (por tanto, no esté probado el enunciado) frente a razones epistémicas suficientes. Contrariamente, se asume que cuando alguien conoce, está persuadido, pero con la particularidad de que su persuasión está sustentada en pruebas (i.e. razones epistémicas) suficientes.

Ahora bien, Taruffo rechaza además la 'prueba como persuasión' por otra razón, a saber: «el juez debe fundar su decisión exclusivamente en pruebas admisibles (...) no es irrelevante cuál sea la fuente de su persuasión: si ésta deriva de informaciones extrajudiciales (la denominada "ciencia privada" del juez) o de pruebas ilícitas o inadmisibles, la decisión no se podrá basar en ellas solo porque el juez las encuentre persuasivas ${ }^{48}$. En este orden de ideas, y volviendo a lo dicho al final del apartado precedente, lo llamativo es que en este pasaje la 'prueba como persuasión' aparece a los ojos de Taruffo como indeseable aun cuando mayormente fundada desde el punto de vista epistémico.

Cabría dilucidar el sentido que en el pensamiento de Taruffo tiene ese deber del juez (i.e. jurídico, moral, etc.), pero cualquiera sea el caso, lo cierto es que el aceptarlo implica relegar el objetivo de máximo acercamiento epistémicamente posible a 'la verdad', en favor del 'respeto de las reglas de juego', considerándose justificado (desde el plano normativo del que provenga ese deber) que las decisiones contengan enunciados fácticos falsos o, como mínimo, contrarios a lo que las (mejores) razones epistémicas disponibles indican como verdadero. Lo llamativo es que si ese deber se concibiese como moral (y si se considerase que lo moralmente correcto es justo), pues decidir sobre la base de las pruebas meramente admisibles (aun cuando epistémicamente 'peores') y tener por probados enunciados fácticos falsos (o viceversa) no sería ni siquiera extrajurídicamente injusto.

${ }^{47}$ Cfr. Taruffo, p. 35.

${ }^{48}$ Taruffo $_{\mathrm{a}}$, p. 34. 


\section{II.2. Motivación, inmediación y control sobre la justificación de los enunciados fácticos}

Suele concebirse a la prueba (sea como persuasión, sea como conocimiento) como especialmente vinculada con la noción de inmediación. Para quienes creen que la prueba ha de dirigirse a persuadir con independencia de toda razón epistémica, la inmediación se presenta como fuente privilegiada de esa irracional e inexpresable convicción ${ }^{49}$. Para quienes creen que la prueba ha de dirigirse a proporcionar razones epistémicas, es usual considerar que la inmediación ofrece un acceso privilegiado a esas razones, que no serían evaluables por quien no comparta esa posición. Esto conduciría a «blindar el juicio y la decisión de primera instancia frente a la crítica en otro plano jurisdiccional; puesto que algunos elementos de convicción, considerados fundamentales para la formación del criterio judicial en tema de hechos, por su difícil o imposible verbalización, por su práctica intraducibilidad en palabras, resultarían infiscalizables» ${ }^{50}$.

La cuestión ha tenido relevancia práctica en diversos sistemas procesales, especialmente en lo relativo al rechazo de ciertos recursos concebidos como limitados a la revisión de 'cuestiones de derecho'. En especial, allí donde esos recursos son los únicos previstos contra determinadas decisiones, al tiempo que (se entiende haya) otras normas, a veces con jerarquía supralegal, que garantizan un recurso integro ${ }^{51}$.

Dejando de lado los argumentos de corte esencialista en relación a ciertas vías impugnativas ${ }^{52}$, como también aquellos que se aferran a la

${ }^{49}$ A esto apuntaban especialmente Gascón Abellán y Taruffo ${ }_{b}$, p. 82.

${ }^{50}$ Andrés Ibañez, p. 59. Véase también Andrés IbÁÑez, Perfecto, "Acerca de la motivación de los hechos en la sentencia penal", Doxa: cuadernos de filosofia del derecho, 12, 1992.

${ }^{51}$ Por ejemplo, en relación al art. 8, 2, h del Pacto de San José de Costa Rica se ha dicho que «[i]ndependientemente de la denominación que se le dé al recurso existente para recurrir un fallo, lo importante es que dicho recurso garantice un examen integral de la decisión recurrida" (CIDH, Herrera Ulloa vs. Costa Rica, 2/7/2004, párrafo 165).

${ }^{52}$ Aludiendo, por ejemplo, a la naturaleza jurídica del recurso (e.g. de casación) como excluyente de la revisión de la justificación de enunciados fácticos. 
Tres discusiones acerca de la relación entre prueba y verdad

letra de ciertas disposiciones y/o a interpretaciones de índole conservadora $^{53}$, muchas veces ese rechazo se ha fundado en una pretendida imposibilidad epistémica de control de la justificación de la premisa fáctica por ser ella, precisamente, producto de la inmediación ${ }^{54}$.

Este punto no ha sido pasado por alto en la 'epistemología jurídica' de los últimos años. Se ha mostrado que ese aparente blindaje epistémico producido por la inmediación, se diluye frente a una explicitación del sustento de cada enunciado fáctico, y distinguiendo, consecuente y cuidadosamente, a los genuinos enunciados de inmediación de aquellos inferenciales: un enunciado solo es producto de la inmediación «cuando su apoyo no está basado o justificado en premisas sino en hechos externos»; se trata de productos de la percepción del individuo que los sostiene y dependen, por tanto, de su posición epistémica en el sentido de 'relación sujeto-mundo'55. Por el contrario, «[u]n enunciado es inferencial cuando es o puede ser reconstruido como una conclusión basada en premisas» de modo que se presenta como una 'relación enunciado-enunciado' donde cada premisa es una conclusión que se infiere de otras premisas ${ }^{56}$.

La imposibilidad de control epistémico afecta pura y exclusivamente a los enunciados de inmediación. Pero lo cierto es que ellos,

${ }^{53}$ Pienso en aquellas interpretaciones que atribuyen significado a ciertas disposiciones que regulan, por ejemplo, el recurso de casación, sobre la base de que la intención del legislador histórico habría sido la de excluir del control en alzada a las 'cuestiones sobre hechos'.

${ }^{54}$ Obstáculo que afectaría a todo recurso de aquellos a que los procesalistas califican como 'de efecto devolutivo'.

55 «Por eso decimos que los enunciados de inmediación son auténticos enunciados "últimos", pues "antes" de ellos no hay otros enunciados sino directamente una percepción sensorial» (PÉREZ BARBERÁ, GABRIEL, "Casación penal y posibilidad de contol. Alcance del fallo "Casal" y del método alemán invocado por la Corte", Pensamiento Penal y Criminológico: revista de Derecho Penal integrado, 11, 2007, p. 247).

${ }^{56}$ Pérez Barberá, Gabriel \& Hernán Bouvier, "Casación, lógica y valoración de la prueba: Un análisis de la argumetación sobre los hechos en las sentencias de los tribunales casatorios", Pensamiento Penal y Criminológico: revista de Derecho Penal integrado, 9, 2004, p. 188. 
cuantitativa y cualitativamente, carecen de la relevancia que históricamente se les atribuyó. En efecto, imagínese al tribunal (o jurado) que recepta la prueba en el juicio oral: ¿qué es producto de la inmediación? El enunciado según el cual el testigo 'movió las manos de tal o cual modo', por ejemplo, pero nada más que eso. Lo que de allí se siga, e.g. 'su nerviosismo', será ya producto de la inferencia y será, por tanto, controlable.

A esto se apuntaba ya en Discusiones 3 cuando se subrayaba la importancia de que el fundamento de los enunciados fácticos contenga «... datos verbalizables y suficientemente verbalizados, para que resulten intersubjetivamente evaluables, susceptibles de un control racional de su valor convictivo mediante la puesta en relación con los de otra procedencia ${ }^{57}$. Así, el espacio de lo incontrolable se reduce considerablemente, desmitificándose a la inmediación, si se desenmascara a los enunciados que, presentados como perceptivos, no son sino enunciados inferenciales con premisas ocultas ${ }^{58}$.

\section{Tercera discusión: presunción de inocencia y estándares de prueba}

Aunque no discutido explícitamente, lo relativo a la suficiencia de las razones epistémicas en favor de enunciados fácticos permeaba cada espacio de Discusiones 3. En la introducción al número, lue-

${ }^{57}$ Andrés Ibañez, p. 59-60. En el mismo sentido AndrÉs IbÁÑEz, PerFecto, "Acerca de la motivación de los hechos en la sentencia penal", Doxa: cuadernos de filosofia del derecho, 12, 1992, p. 297-299. La Corte Suprema de Justicia de la Nación se enfrentó a esta cuestión en varias ocasiones, por todos véase "Casal, Matias E., y otros s/ robo simple en grado de tentativa" (causa $N^{\circ} 1681$, del 20/09/2005).

58 Véase, Pérez Barberá, Gabriel \& Hernán Bouvier, "Casación, lógica y valoración de la prueba: Un análisis de la argumetación sobre los hechos en las sentencias de los tribunales casatorios", Pensamiento Penal y Criminológico: revista de Derecho Penal integrado, 9, 2004, p. 187. La 'magnificación de lo que es producto de la inmediación' se basa también en la sospechosa distinción entre prueba directa y prueba indirecta que por razones de extensión no puede ser aquí abordada. 
Tres discusiones acerca de la relación entre prueba y verdad

go de identificarse como centrales a las cuestiones del 'concepto y finalidad de la prueba', del 'objeto de prueba', de la 'valoración de la prueba' y de la 'motivación de la declaración de hechos probados', se presentaba a tres de ellas como indisolublemente ligadas a la suficiencia: en efecto, se decía que 'concepto y finalidad' de la prueba, han de determinar «cuándo es razonable sostener que un enunciado parece verdadero, a la luz de la información de que disponemos» ${ }^{59}$; que las reflexiones acerca de la 'valoración' habrían de dirigirse «a obtener criterios que nos permitan determinar cuándo un enunciado está probado, es decir, cuándo es razonable considerar que ese enunciado es verdadero, a la luz de la información de la que disponemos». En lo relativo a la 'motivación de la declaración de hechos probados', por último, se decía que ella concierne a «... los instrumentos para mostrar (hacia terceros) las razones que justifican dicha declaración ${ }^{60}$.

La omnipresencia de la cuestión de la suficiencia probatoria y, con ella, de los estándares de prueba ${ }^{61}$, parece insoslayable toda vez que se reconozca que la justificación del enunciado fáctico de la decisión judicial depende de un razonamiento no concluyente; en términos generales, una inferencia inductiva cuya justificación no puede ser sino probabilística y falible ${ }^{62}$. Esto, en conjunción con la necesidad jurídica de decidir (contracara de la prohibición de denegar justicia) declarando 'probado' o 'no probado' al enunciado correspondiente. La com-

${ }^{59}$ Ferrer Beltrán, Jordi \& Daniel González Lagier, "Introducción", Discusiones, 3, 2003, p. 8.

${ }^{60}$ Ibid., p. 9.

${ }^{61} \mathrm{La}$ expresión 'estándares de prueba' aparece, sin embargo, recién al final del número: Taruffo, p. 93. Recientemente se ha planteado una especie de paralelismo entre estándares de prueba (pretendidamente anglosajones) y sistemas de valoración (pretendidamente latinos): LLUCH, XAVIER ABEL, "La dosis de prueba: entre el common law y el civil law", Doxa: Cuadernos de Filosofia del Derecho, 35, 2012. Creo, por el contrario, que todo sistema de valoración requiere de un criterio de suficiencia (i.e. estándar) y que todo estándar supone un sistema de valoración. No puedo ocuparme de esto aquí, aunque parte de las razones que sustentan esa posición quedarán expuestas en $\S 3.1$.

62 Por todos, Ferrajoli, Luigi, Derecho y razón: teoría del garantismo penal, Madrid, Trotta, 2005, p. 53. 
binación de inferencia probabilística y necesidad de decidir conduce inexorablemente a la búsqueda de criterios de suficiencia concretos: estándares de prueba.

La cuestión ocuparía un lugar central en la agenda epistemológico-jurídica de los años sucesivos a Discusiones 3. Sin embargo, la epistemología empezaría a mostrar sus limitaciones. En particular, su relativa falta de idoneidad para ofrecer unidades de medida de la prueba, y su consecuente impotencia para determinar criterios de suficiencia concluyentes.

\section{III.1. E1 peso de la prueba y los límites de la epistemología}

El contar con un criterio de suficiencia probatoria depende lógicamente de la determinación previa de un método que suministre las unidades de medida sobre cuya base formularlo. En la búsqueda de tales unidades de medida, la 'epistemología jurídica' se ha centrado en general en la noción de probabilidad en cuyo contenido, se supone, ellas habrían de encontrarse.

Los autores se han dividido según consideren o no posible, a estos efectos, recurrir a las nociones de probabilidad que responden al cálculo matemático ${ }^{63}$. Entre los primeros, dos líneas de pensamiento han tenido particular repercusión: (i) la que concibe a la probabilidad como frecuencia relativa de eventos ${ }^{64}$; $\mathrm{y}$ (ii) la que la concibe como grado

63 Por todos, Cohen, L. Jonathan, Introduzione alla Filosofia dell'induzione e della probabilità, Milano, Giuffrè, 1998 [1989]. El que la probabilidad sea matemáticamente calculable significa que le son aplicables las reglas de: (i) complementariedad para la negación (i.e. la conjunción de la probabilidad de una proposición y su negación suman siempre 1), y (ii) multiplicación para la conjunción (i.e. la probabilidad de que dos proposiciones sean verdaderas es igual a la multiplicación de la probabilidad de cada una de ellas).

${ }^{64}$ Téngase presente que una cosa es la prueba estadística y otra distinta la concepción de los estándares de prueba en términos estadísticos (PARDO, Michael S., Estándares de prueba y teoría de la prueba, en VÁzQuez, CÁrmen (ED.), Estándares de prueba y prueba científica, Madrid - Barcelona Buenos Aires - Sao Paulo, Marcial Pons, 99-118, 2013, p. 104). Una defensa de la utilización de estadísticas en general en el marco jurídico en ScHAUER, 
Tres discusiones acerca de la relación entre prueba y verdad

de creencia proclamando además la aplicación del teorema de Bayes al razonamiento judicial sobre hechos ${ }^{65}$.

En cualquier caso, estas tentativas han resultado en general infructuosas en el marco de la decisión judicial por varias razones, pero principalmente por la inaplicabilidad de las reglas del cálculo matemático dados ciertos resultados contraintuitivos y/o jurídicamente inaceptables a que conduciría ${ }^{66}$.

En contraste, suele entenderse hoy a la probabilidad como soporte lógico que la evidencia ofrece a la hipótesis desde una perspectiva 'baconiana', no susceptible de someterse al cálculo matemático ${ }^{67}$. A muy grandes rasgos, se ha sostenido en esta orientación, que la 'probabilidad' equivale al apoyo inductivo función de la variedad de variables

FREDERICK, Di ogni erba un fascio: generalizzazioni, profili, stereotipi nel mondo della giustizia, Bologna, Il Mulino, 2008, p. 89. Algunas críticas a estas posiciones en CoHEn, L. Johathan, The probable and the provable, Oxford, Clarendon Press, 1977, p. 74, Ferrer Beltrán, Jordi, La valoración racional de la prueba, Madrid, Marcial Pons, 2007, p. 98, Gascón Abellán, Marina, Los hechos en el derecho: Bases argumentales de la prueba, Madrid- Barcelona, Marcial Pons, 2004, p. 198.

${ }^{65}$ Por todos, De FinetTI, BRuno, Bayesianesimo: il suo ruolo unificante per i fondamenti e le applicazioni della statistica, en Monari, PaOla \& Daniela CocchI, Probabilità e induzione, Bologna, Cooperativa Libraria Universitaria Editrice Bologna, 205-228, 1993 [1973], p. 205. Conviene tener presente que el nivel de discurso que los defensores del método bayesiano asumen es explícitamente normativo (por todos, LINDLEY, DENNIS V., Making decision, London, Wiley Interscience, 1971, p. 3-4). Ver también, GARbolino, PAOLO, I Fatti e le opinioni: la moderna arte della congettura, Roma, Laterza, 1997, p. 93-98. Una serie de trabajos dirigidos explícitamente a la aplicación del teorema de Bayes a la decisión judicial en Tillers, Peter, Eric D. Green \& Alberto Mura, L'Inferenza probabilistica nel diritto delle prove: usi e limiti del bayesianesimo, Milano, Giuffrè, 2003. Por razones de extensión, estas ideas no pueden ser aquí ampliadas.

${ }^{66}$ Por todos, Cohen, L. Johathan, The probable and the provable, Oxford, Clarendon Press, 1977, p. 58-91, CoHEN, L. JonAthan, Introduzione alla Filosofia dell'induzione e della probabilità, Milano, Giuffrè, 1998 [1989], p. 185-186.

${ }^{67}$ Cohen, L. Johathan, The probable and the provable, Oxford, Clarendon Press, 1977, p. 121. Cfr. Hempel, Carl Gustav, La explicación cientifica: estudios sobre la filosofia de la ciencia, Barcelona, Paidos, 2005 [1965], p. 16. 


\section{Diego Dei Vecchi}

a que una hipótesis es sometida ${ }^{68}$. A mayor complejidad de variables que la hipótesis supera mayor será su apoyo inductivo. Pero sea como fuere, lo cierto es que el método no proporciona instrumentos para cuantificar el peso probatorio de cada hipótesis en competencia, sirviendo tan solo para compararlas, para ordenarlas ${ }^{69}$.

En estas circunstancias se ha recurrido a nociones emparentadas con la llamada «inferencia hacia la mejor explicación» ${ }^{70}$. Se sostiene que estas nociones serían adecuadas tanto para describir la práctica judicial en lo relativo a la aceptación de enunciados fácticos cuanto para proporcionar criterios normativos de comparación y selección de hipótesis fácticas 'probablemente verdaderas' ${ }^{71}$. Pero en todo caso, los criterios podrán determinarse solo sobre la base del peso comparativo de las inferencias y sus conclusiones. Y esto sería todo cuanto la epistemología puede ofrecer ${ }^{72}$.

${ }^{68}$ Cohen, Jonathan L., "Twelve Questions about Keynes's Concept of Weight", British Journal for the Philosophy of Science, 37, 1985. Esta noción parece estar subyacente muchas veces en los conceptos de razonabilidad, verosimilitud, confirmabilidad, credibilidad, fiabilidad o plausibilidad muchas veces utilizados al referir a la justificación de la premisa fáctica de la decisión judicial (cfr. por ejemplo, Asencio Mellado, José María, Prueba prohibida y prueba preconstituida, Madrid, Trivium, 1989, p. 16, FERRAJOLI, Luigi, Derecho y razón: teoría del garantismo penal, Madrid, Trotta, 2005, p. 120, GonzÁlez Lagier, Daniel, Quaestio facti: ensayos sobre prueba, causalidad y acción, Lima-Bogotá, Palestra-Temis, 2005, p. 66, 72).

${ }^{69}$ Ferrer BeLtrán, Jordi, La valoración racional de la prueba, Madrid, Marcial Pons, 2007, p. 122.

${ }^{70}$ Por todos, Harman, Gilbert H., "The inference to the Best Explanation", The Philosophical Review, 74, 1965.

${ }^{71}$ Canale, Damiano, Il ragionamento giuridico, en Pino, Giorgio, Aldo Schiavello \& Vittorio Villa, Filosofia del diritto. Introduzione critica al pensiero giuridico e al diritto positivo (Estratto), Torino, Giappichelli, 195230, 2013, p. 225, Pardo, Michael S. \& Ronald J. Allen, "Juridical proof and the best explanation", Law and Philosophy, 27 3, 2008, PARdo, MichaEL S., Estándares de prueba y teoría de la prueba, en CÁRMEN (ED.) VÁzQuez, Estándares de prueba y prueba científica, Madrid - Barcelona - Buenos Aires - Sao Paulo, Marcial Pons, 99-118, 2013.

72 Bayón Mohino, JuAn CARLos, "Epistemología, moral y prueba de los hechos: hacia un enfoque no benthamiano", Analisi e diritto 2008, 2009, p. 24. 
Tres discusiones acerca de la relación entre prueba y verdad

\section{III.2. La prueba suficiente y la presunción de inocencia}

La limitada oferta de la epistemología, i.e. la de determinar cuál es la mejor hipótesis entre las que están en competencia, no es de por sí despreciable. Sin embargo, desde el punto de vista axiológico, solo permite satisfacer la que se calificó como «sensibilidad mínima» al riesgo de error, esto es, la que se conforma con tener por probada siempre la mejor hipótesis, incluso siendo esa una mala hipótesis, o siendo solo mínimamente mejor que la que le sigue en el orden de bondad. De tal modo y al menos en principio, los riesgos de error (en términos procesal-penalistas, las falsas condenas y las falsas absoluciones) se distribuirían por igual ${ }^{73}$.

No obstante, se afirma que el derecho tiene, o que debe tener, una «sensibilidad más que mínima» al riesgo de error; por lo menos en ciertos ámbitos disciplinares (e.g. el penal), donde no sería suficiente con determinar la mejor hipótesis, sino que sería necesario contar con una hipótesis especialmente buena, tal que se reduzcan 'más que mínimamente' ciertos errores (e.g. los de condenas a inocentes).

En este orden de ideas, la medida de bondad probatoria que una hipótesis deba satisfacer depende de un juicio valorativo que determina cuándo es justa la distribución del riesgo de error. En la búsqueda de esta medida, prioritariamente enfocada al ámbito del proceso penal, se ha recurrido usualmente a la 'presunción de inocencia', como presunta expresión de un juicio valorativo determinativo de la suficiencia de las pruebas para condenar. Específicamente, se trataría de una valoración subyacente a la 'regla de juicio' que históricamente se ha derivado de dicha presunción ${ }^{74}$, y que suele identificarse con el in dubio pro

${ }^{73}$ Ibid., p. . Las expresiones «sensibilidad mínima» y «más que mínima» son del autor.

${ }^{74}$ Además de esta regla, usualmente se derivan de la presunción de inocencia una regla 'de tratamiento' del imputado como inocente y otra de 'producción de la prueba' (carga -o más precisamente 'responsabilidad'de la acusación). Cfr. Fernández López, Mercedes, Prueba y presunción de inocencia, Madrid, Iustel, 2005, p. 139, PAUlesu, PIER PAolo, La presunzione di non colpevolezza dell'imputato, 30, Torino, G. Giappichelli, 2009, p. 10. 
reo y/o con la conocida como prueba más allá de toda duda razonable ${ }^{75}$. En cualquier caso, se trataría de una preferencia de las absoluciones a culpables por sobre las condenas a inocentes ${ }^{76}$.

Sin embargo, el recurso a estos enunciados y reglas se ha mostrado recientemente como infructuoso ${ }^{77}$. Esto así porque de la proscripción de condena ante la duda o la duda razonable, nada se sigue respecto al criterio de suficiencia, al menos no si se rechazan los criterios probatorios meramente internos, i.e. a la 'mera persuasión'. En efecto, en este último sentido, la duda excluye, por definición, creencia, convicción y (mera) persuasión; pero dado que ni la presencia ni la ausencia de esos estados subjetivos podría determinar que un enunciado fáctico en el ámbito judicial esté probado, la presencia o no de dudas (así entendidas) tampoco proporciona criterio alguno.

Decía recientemente Ferrer Beltrán que 'duda' puede aquí entenderse en al menos tres sentidos: (i) como estado psicológico excluyente de los otros estados antes referidos; (ii) como imposibilidad racional de certeza y (iii) como contrario a suficientemente probado. Dado que (i) conduce a los problemas aludidos al hablar de prueba como mera persuasión y que (ii) conduciría a que ningún enunciado fáctico pueda considerarse probado jamás (dado que esas dudas son insoslayables ante razonamiento aproximativos), entonces solo resta (iii). Pero entonces, la 'ausencia de duda' es función del estándar de prueba (y no

${ }^{75}$ Así Andrés Ibañez, p. 61, Taruffo, p. 92. Estos aspectos 'probatorios' de la presunción de inocencia, se dice, son prioritarios en el common law respecto a la 'regla de trato' (cfr. Batia, Giovanna \& Alessandro Pizzo, "La tutela dell' imputato - Saggio storico - concettuale", Diritto.it, 2011).

${ }^{76}$ Naturalmente, 'culpable' e 'inocente' aquí son utilizados en sentido 'material' sin presuponer 'declaración de culpabilidad' (cfr. LAUDAN, LARRY, "The Presumption of Innocence: Material or Probatory?", Legal Theory, 11, 2005).

${ }^{77}$ Larry Laudan, ha ido criticando con creciente fervor la 'razonabilidad' del beyond a rasonable doubt en varios sentidos, entre ellos, incluida la valoración subyacente antes mencionada (en especial LAUDAN, LARRY, "Is reasonable doubt reasonable?", Legal Theory, 9, 2003, p. 331). Ver también, Allen, RONALD J. \& LARRY LAUDAN, "Deadly dilemmas", Texas Tech Law Revew, 41, 2008, Laudan, Larry \& Ronald J. Allen, "Deadly Dilemmas II: Bail and crime", Chicago Kent Law Review, 85, 2010. 
al revés), de modo que la presunción de inocencia y sus reglas derivadas son compatibles con diversos estándares, presuponiendo alguno, pero siendo insignificantes para determinarlo ${ }^{78}$.

Más aún, se ha dicho recientemente que quienes defienden el juicio valorativo pretendidamente subyacente a la presunción de inocencia, según el cual es preferible la absolución del culpable que la condena del inocente, no cuentan con una herramienta conceptual para decir cuánto peor o mejor es uno u otro resultado, y guiar así la formulación de un estándar ${ }^{79}$. En consecuencia, incluso si la epistemología proporcionase criterios cardinales del peso de las hipótesis (y no meramente ordinales), todavía habría que buscar criterios valorativos que determinasen cuán 'cardinalmente apoyada' debe de estar una hipótesis para la adopción de tal o cual decisión.

\section{Consideraciones finales}

Algunos años más tarde de la aparición de Discusiones 3, en la que se lamentaba la histórica marginalización de las cuestiones epistemológicas sobre la prueba en el proceso judicial, decía Juan Carlos Bayón que «decididamente, y por fortuna, ese tiempo ha pasado ya» ${ }^{80}$. No solo se ha multiplicado la literatura sobre el asunto, sino que además se ha iniciado y mantenido un fecundo, y acaso inusual, diálogo entre autores de diversas tradiciones jurídicas así como entre filósofos, epistemólogos y juristas.

Sin embargo, especialmente en lo relativo a los estándares de prueba, la brecha entre teoría y práctica parece haberse más bien ampliado, tanto por lo inadecuado de las tentativas explicativas, cuanto por

${ }^{78}$ FerRer BeLtRÁN, JoRDI, Una concepción minimalista y garantista de la presunción de inocencia, Girona, Universitat de Girona, 2010, p. 285-295.

${ }^{79}$ Por todos, Laudan, Larry, The Rules of Trial, Political Morality, and the Costs of Error: Or, Is proof Beyond a Reasonable Doubt Doing More Harm than Good?, en Green, Leslie \& Brian Leiter, Oxford studies in philosophy of law, 1, Oxford, Oxford University Press, 195-227, 2011.

${ }^{80}$ BAYÓn Mohino, JuAn CARLOS, "Epistemología, moral y prueba de los hechos: hacia un enfoque no benthamiano", Analisi e diritto 2008, 2009, p. 16. 
la implausibilidad de las propuestas normativas ${ }^{81}$. Al tiempo que se pretende (o se pretende se deba) ser «más que mínimamente sensible» al riesgo de error, se ha llegado a la aparentemente indefectible conclusión de que, no solo la epistemología es inidónea para satisfacer tal pretensión, sino que además los enunciados jurídicos son inexpresivos de una medida de «sensibilidad» moral en concreto, e incluso que la filosofía moral estaría en dificultades para proporcionar criterios de distribución. A pesar de esto, los jueces continúan diariamente, y quizás solo porque no pueden no decidir, declarando suficientemente probados los enunciados fácticos de sus decisiones. En estas circunstancias, la real distribución del riesgo de error correspondiente a esa suficiencia, pareciera ser más bien función de la valoración de cada decisor en cada caso ${ }^{82}$. De tal modo, 'la prueba como conocimiento' corre el peligro de caer, sin quererlo, en 'mera persuasión', al menos en el sentido de persuasión de haber prueba moralmente suficiente. Esto ha llevado a muchos de quienes propician que las decisiones morales relevantes sean adoptadas democráticamente, a proponer la revisión de los sistemas de valoración de la prueba que conducen a una situación tal, a modo de retorno a los sistemas legales ${ }^{83}$.

Sea como fuere, lo cierto es que el razonamiento probatorio de la decisión judicial está siendo íntegramente puesto a prueba, especialmente en lo relativo a los criterios de suficiencia. La discusión parece hoy estar virando desde la epistemología a la moral. Pero lo cierto es que en todo caso, no parece poder afirmarse todavía que haya prueba suficiente.

${ }^{81}$ En este orden de ideas, Allen, Ronald J., Los estándares de prueba y los límites del análisis jurídico, en VÁzQuez, CÁRMEN (ED.), Estándares de prueba y prueba científica, Madrid - Barcelona - Buenos Aires - Sao Paulo, Marcial Pons, 41-64, 2013, p. 56-57.

${ }^{2}$ Stein, Alex, Foundations of evidence law, Oxford - New York, Oxford University Press, 2005, p. 119. Ciertamente, no se sigue de esto que esas valoraciones no puedan apoyarse en razones, razones prácticas, claro está.

83 Distintas propuestas en esta orientación, y con diversos matices, en BAYÓN Mohino, JUAN CARLOS, "Epistemología, moral y prueba de los hechos: hacia un enfoque no benthamiano", Analisi e diritto 2008, 2009, SteIn, Alex, Foundations of evidence law, Oxford - New York, Oxford University Press, 2005. 
Tres discusiones acerca de la relación entre prueba y verdad

\section{Bibliografía}

Allen, R. J., Los estándares de prueba y los límites del análisis jurídico, en C. e. Vázquez, Estándares de prueba y prueba científica, Madrid Barcelona - Buenos Aires - Sao Paulo, Marcial Pons, 41-64, 2013. Allen, R. J. \& L. Laudan, "Deadly dilemmas", Texas Tech Law Revew, 41, 2008.

Anderson, T., W. Twining \& D. A. Schum, Analysis of evidence, New York, Cambridge University Press, 2005 [1991].

ANDrÉs IbÁÑEz, P., "Acerca de la motivación de los hechos en la sentencia penal", Doxa: Cuadernos de Filosofia del Derecho, 12, 1992.

ANDRÉs IBÁÑEZ, P., "Sobre prueba y proceso penal", Discusiones, 3, 2003.

Asencio Mellado, J. M., Prueba prohibida y prueba preconstituida, Madrid, Trivium, 1989.

Batia, G. \& A. Pizzo, "La tutela dell' imputato - Saggio storico - concettuale", Diritto.it, 2011.

BAYón Mohino, J. C., "Epistemología, moral y prueba de los hechos: hacia un enfoque no benthamiano", Analisi e diritto 2008, 2009.

Bouzat, A. \& A. S. CANTARo, "Verdad y prueba en el proceso acusatorio", Discusiones, 3, 2003.

Briseño Sierra, H., Derecho procesal, México, D.F, Harla, 1995.

Canale, D., Il ragionamento giuridico, en G. Pino, A. Schiavello \& V. Villa, Filosofia del diritto. Introduzione critica al pensiero giuridico e al diritto positivo (Estratto), Torino, Giappichelli, 195-230, 2013.

CARAcciolo, R., El problema de los hechos en la justificación de la sentencia, Universidad Nacional del Sur, 2000.

CARAcciolo, R. A., Justificación normativa y pertenencia, en R. A. Caracciolo, El derecho desde la filosofía: ensayos, Madrid, Centro de Estudios Politicos y Constitucionales, 37-69, 2009.

Cohen, J. L., "Twelve Questions about Keynes's Concept of Weight", British Journal for the Philosophy of Science, 37, 1985.

CoHEn, L. J., The probable and the provable, Oxford, Clarendon Press, 1977. Cohen, L. J., Introduzione alla Filosofia dell'induzione e della probabilità, Milano, Giuffrè, 1998 [1989].

Couture, E. J., Fundamentos de Derecho Procesal Civil, Buenos Aires, Depalma, 1958. 
DAMAŠKA, M. R., Il Diritto delle prove alla deriva, Bologna, il Mulino, 2003. DE FINETTI, B., Bayesianesimo: il suo ruolo unificante per i fondamenti e le applicazioni della statistica, en P. Monari \& D. Cocchi, Probabilità e induzione, Bologna, Cooperativa Libraria Universitaria Editrice Bologna, 205-228, 1993 [1973].

ENGEL, C., "A behavioral perspective on a conflict between american and continental european law", Vermont Law Review, 33, 2009.

Fernández López, M., Prueba y presunción de inocencia, Madrid, Iustel, 2005.

Ferrajoli, L., Derecho y razón: teoría del garantismo penal, Madrid, Trotta, 2005.

Ferrer Beltrán, J., 'Está probado que p', en L. Triolo, Prassi giuridica e controllo di razionalità, I, Torino, G. Giappichelli, 73-96, 2001.

Ferrer Beltrán, J., Prueba y verdad en el derecho, Madrid, Marcial Pons, 2002.

FERRER BELTRÁn, J., La valoración racional de la prueba, Madrid, Marcial Pons, 2007.

FerRer BeLtrÁN, J., Una concepción minimalista y garantista de la presunción de inocencia, Girona, Universitat de Girona, 2010.

Ferrer Beltrán, J., La valutazione razionale della prova, Milano, Giuffrè, 2012.

Ferrer Beltrán, J. \& D. GonzÁlez Lagier, "Introducción", Discusiones, 3, 2003.

FERruA, P., Il giudizio penale: fatto e valore giuridico, en A.A.V.V., La prova nel dibattimento penale, Torino, G. Giappichelli, 317-410, 2007.

Fiss, O. M., "The Social and Political Foundations of Adjudication", Law and Human Behavior, 6 2, 1982.

Garbolino, P., I Fatti e le opinioni: la moderna arte della congettura, Roma, Laterza, 1997.

Gascón Abellán, M., "Concepciones de la prueba. Observación a propósito de Algunas consideraciones sobre la relación entre prueba y verdad, de Michele Taruffo", Discusiones, 3, 2003.

Gascón Abellán, M., Los hechos en el derecho: Bases argumentales de la prueba, Madrid- Barcelona, Marcial Pons, 2004.

GonzÁlez Lagier, D., Quaestio facti: ensayos sobre prueba, causalidad y acción, Lima-Bogotá, Palestra-Temis, 2005. 
GonzÁlez Lagier, D., "Hechos y conceptos", Dialnet: cuadernos electrónicos de filosofia del derecho, 15, 2007.

GuAstini, R., Interpretare e argomentare, Milano, Giuffrè, 2011.

HaRman, G. H., "The inference to the Best Explanation", The Philosophical Review, 74, 1965.

Hempel, C. G., La explicación científica: estudios sobre la filosofia de la ciencia, Barcelona, Paidos, 2005 [1965].

LAUDAN, L., "Is reasonable doubt reasonable?", Legal Theory, 9, 2003.

LAUDAN, L., "The Presumption of Innocence: Material or Probatory?", Legal Theory, 11, 2005.

Laudan, L., The Rules of Trial, Political Morality, and the Costs of Error: Or, Is proof Beyond a Reasonable Doubt Doing More Harm than Good?, en L. Green \& B. Leiter, Oxford studies in philosophy of law, 1, Oxford, Oxford University Press, 195-227, 2011.

LaudAn, L. \& R. J. Allen, "Deadly Dilemmas II: Bail and crime", Chicago Kent Law Review, 85, 2010.

Lindley, D. V., Making decision, London, Wiley Interscience, 1971.

Lluch, X. A., "La dosis de prueba: entre el common law y el civil law", Doxa: Cuadernos de Filosofia del Derecho, 35, 2012.

Maier, J. B., Derecho Procesal Penal: Fundamentos, 1, Buenos Aires, del Puerto, 2004.

Mazzarese, T., Forme di razionalità delle decisioni giudiziali, Torino, Giappichelli, 1996.

PARDo, M. S., Estándares de prueba y teoría de la prueba, en C. e. Vázquez, Estándares de prueba y prueba científica, Madrid - Barcelona Buenos Aires - Sao Paulo, Marcial Pons, 99-118, 2013.

PARDo, M. S. \& R. J. Allen, "Juridical proof and the best explanation", Law and Philosophy, 27 3, 2008.

Paulesu, P. P., La presunzione di non colpevolezza dell'imputato, 30, Torino, G. Giappichelli, 2009.

Pérez BarberÁ, G., "Casación penal y posibilidad de contol. Alcance del fallo "Casal" y del método alemán invocado por la Corte", Pensamiento Penal y Criminológico: revista de Derecho Penal integrado, 11, 2007.

Pérez Barberá, G. \& H. Bouvier, "Casación, lógica y valoración de la prueba: Un análisis de la argumetación sobre los hechos en 
las sentencias de los tribunales casatorios", Pensamiento Penal y Criminológico: revista de Derecho Penal integrado, 9, 2004.

Redondo, M. C., "Argumentación auténtica y argumentación estratégica", Revista Brasileira de Filosofia, 238 1, 2012.

SÁnchez Brígido, R. \& H. O. Seleme, "Justificación sin verdad", Analisi e diritto 2001, 2002.

Schauer, F., Di ogni erba un fascio: generalizzazioni, profili, stereotipi nel mondo della giustizia, Bologna, Il Mulino, 2008.

Stein, A., Foundations of evidence law, Oxford - New York, Oxford University Press, 2005.

TARUFFo, M., La prova dei fatti giuridici: nozioni generali, Milano, Giuffre, 1992.

TARufFo, M., "Algunos comentarios sobre la valoración de la prueba", Discusiones, 3, 2003.

TARUFFo, M., "Algunas consideraciones sobre la relación entre prueba y verdad", Discusiones, 3, 2003.

TARUfFo, M., La semplice verità: il giudice e la costruzione dei fatti, Bari, Laterza, 2009.

TARuffo, M., Consideraciones sobre prueba y motivación, en M. Taruffo, P. Andrés Ibáñez \& A. Candau Pérez, Consideraciones sobre la prueba judicial, Madrid, Fundación Coloquio Juridico Europeo, 17-46, 2010.

Taruffo, M., P. Andrés Ibáñez \& A. Candau Pérez, Consideraciones sobre la prueba judicial, Madrid, Fundación Coloquio Jurídico Europeo, 2010.

Tillers, P., E. D. Green \& A. Mura, L'Inferenza probabilistica nel diritto delle prove: usi e limiti del bayesianesimo, Milano, Giuffrè, 2003.

UBERTIS, G., Fatto e valore nel sistema probatorio penale, Milano, Giuffrè, 1979.

VARGAS, A. L., "Cargas probatorias dinámicas, sus perfiles actuales y algunas respuestas para sus críticos", Activismo y garantismo procesal: Ediciones de la Academia Nacional de Derecho y Cs. Sociales de Córdoba, XL 7, 2009.

WRÓBLEWSKI, J., "Il modello teorico dell'applicazione della legge", Rivista internazionale di Filosofia del Diritto, 46, 1967. 